\title{
Exercise training alters the genomic response to acute exercise in human adipose tissue
}

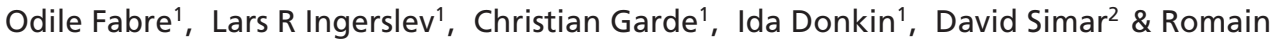 \\ Barrès*,1 \\ ${ }^{1}$ Integrative Physiology, The Novo Nordisk Foundation Center for Basic Metabolic Research, Faculty of Health \& Medical Sciences, \\ University of Copenhagen, Copenhagen, Denmark \\ ${ }^{2}$ Mechanisms of Disease \& Translational Research, School of Medical Sciences, University of New South Wales, Sydney, Australia \\ *Author for correspondence: barres@sund.ku.dk
}

\begin{abstract}
Aim: To determine the genomic mechanisms by which adipose tissue responds to acute and chronic exercise. Methods: We profiled the transcriptomic and epigenetic response to acute exercise in human adipose tissue collected before and after endurance training. Results: Although acute exercises were performed at same relative intensities, the magnitude of transcriptomic changes after acute exercise was reduced by endurance training. DNA methylation remodeling induced by acute exercise was more prominent in trained versus untrained state. We found an overlap between gene expression and DNA methylation changes after acute exercise for 32 genes pre-training and six post-training, notably at adipocyte-specific genes. Conclusion: Training status differentially affects the epigenetic and transcriptomic response to acute exercise in human adipose tissue.
\end{abstract}

First draft submitted: 19 March 2018; Accepted for publication: 11 April 2018; Published online: 19 April 2018

Keywords: adipose tissue $\bullet$ DNA methylation $\bullet$ epigenetics $\bullet$ exercise $\bullet$ human $\bullet$ mRNA $\bullet$ transcriptomic

Adipose tissue is a prominent energy-storing tissue and an important source of fatty acids during aerobic exercise. A single bout of aerobic exercise activates a variety of signals (including adrenergic factors and insulin) that stimulate adipose tissue blood flow and fat mobilization through lipolysis, supporting fatty acids delivery to skeletal muscles [1,2]. After repeated bouts of physical exercise, or exercise training, adipose tissue demonstrates metabolic adaptation, by improving the response to catecholamines, increasing lipolysis and therefore the release of lipids into the circulation $[3,4]$. Such adaptation is associated with marked gene expression changes, in particular for genes driving improved metabolic activity and efficiency through increased oxidative phosphorylation [5,6]. Many of the exercise-induced gene expression changes in adipose tissue function persist for hours after exercise has ceased; in fact, a substantial lipolysis and nonesterified fatty acid release from adipose tissue into the circulation begins about $1 \mathrm{~h}$ postexercise and continues for more than $3 \mathrm{~h}$ [7]. Regular beneficial changes occurring within adipose tissue with exercise training, notably the regulation of fat mass due to enhanced fat mobilization in response to each acute exercise bout, may not only contribute to modulate adipose tissue physiology, but also have downstream consequences for other tissues (e.g., lipid oxidation or esterification in muscle and lipoprotein synthesis in liver), thereby improving whole-body metabolism [8]. The net benefit of exercise training may thus be a reflection of chronic sustained changes induced by each exercise bout and that could be observed at rest. Yet, the mechanism by which such transient exercise-induced adaptive events drive positive changes in adipose tissue remains unclear.

DNA methylation is a major epigenetic process in the genome of eukaryotic cells, which influences gene expression by modulating the access of the transcription machinery to the chromatin or by controlling the degree of chromatin compaction through the recruitment of methyl-binding proteins [9]. The common appreciation is that DNA methylation is stable, which is illustrated by a steady alteration of the DNA methylation blueprint in many diseases [10-13]. In subcutaneous white adipose tissue (sWAT) biopsies from healthy men with a previous low level of physical activity, a 6-month exercise intervention induced global as well as gene-specific DNA methylation changes, including RALBP1, HDAC4 and NCOR2, all involved in the regulation of adipogenesis and glucose or lipid metabolism [14]. DNA methylation can also be a very dynamic process even in somatic, nondividing cells [15,16]. 


\begin{tabular}{|c|c|c|c|}
\hline Clinical characteristic & Pretraining & Post-training & p-value \\
\hline BMI & $22.8 \pm 0.6$ & $22.5 \pm 0.6$ & 0.74 \\
\hline Waist/hip ratio & $0.92 \pm 0.01$ & $0.88 \pm 0.01$ & 0.0003 \\
\hline $\mathrm{VO}_{2} \max \left(\mathrm{ml} \mathrm{O}_{2} / \mathrm{min} / \mathrm{kg}\right)$ & $46.88 \pm 1.22$ & $54.97 \pm 1.17$ & 0.00005 \\
\hline Plasma glucose level (mmol/l) & $4.82 \pm 0.09$ & $4.74 \pm 0.11$ & 0.58 \\
\hline Plasma insulin level ( $\mathrm{mmol} / \mathrm{l})$ & $54.33 \pm 4.89$ & $54.13 \pm 4.08$ & 0.98 \\
\hline $\mathrm{HOMA}_{\mathrm{IR}}$ & $1.96 \pm 0.21$ & $1.89 \pm 0.14$ & 0.77 \\
\hline Estimated average glucose from $\mathrm{HbA} 1 \mathrm{c}(\mathrm{mmol} / \mathrm{l})$ & $5.69 \pm 0.11$ & $5.55 \pm 0.10$ & 0.34 \\
\hline C-reactive protein $(\mathrm{mg} / \mathrm{l})$ & $3.07 \pm 1.18$ & $1.00 \pm 0.00$ & 0.09 \\
\hline Leukocytes (number $/ \mathrm{mm}^{3}$ ) & $6493 \pm 440$ & $5867 \pm 310$ & 0.26 \\
\hline Triglycerides (mmol/l) & $1.09 \pm 0.10$ & $1.11 \pm 0.11$ & 0.92 \\
\hline Total cholesterol (mmol/l) & $4.60 \pm 0.25$ & $4.43 \pm 0.27$ & 0.65 \\
\hline HDL-chol (mmol/l) & $1.36 \pm 0.06$ & $1.45 \pm 0.05$ & 0.32 \\
\hline LDL-chol (mmol/l) & $2.69 \pm 0.24$ & $2.68 \pm 0.22$ & 0.98 \\
\hline
\end{tabular}

Muscle contraction remodels DNA methylation in skeletal muscle cells via gene-specific DNA demethylation [17,18]. Skeletal muscle-specific promoter methylation of genes involved in mitochondrial function, including PPARGC1A, TFAM, MEF2A and PDK4 decreased immediately after a high-intensity exercise bout, with a concomitant increase in the corresponding transcript levels [17]. Thus, physical exercise can remodel DNA methylation in skeletal muscle both acutely and in the long-term [19], thereby modifying gene expression in a tissue-specific manner [20,21]. However, DNA methylation changes induced by acute exercise and potential impact on gene expression have not yet been investigated in adipose tissue.

In the present study, we aimed to determine the epigenetic and transcriptomic mechanisms by which adipose tissue responds to acute and chronic exercise. We recruited 15 sedentary male participants who performed an acute exercise bout before and after a 6-week endurance-training program. Using RNA sequencing (RNA-seq) and reduced representation bisulfite sequencing (RRBS), we analyzed mRNA expression and DNA methylation in human sWAT biopsies collected at various time points before and after the acute exercise bouts. Our findings provide insight into the role of DNA methylation in the transcriptional response to exercise in adipose tissue.

\section{Patients \& methods}

\section{Study participants}

Fifteen male volunteers, aged between 19 and $27(22.9 \pm 0.5)$ years old, were recruited to participate in the study. A portion of this cohort has previously been described [18]. All were healthy, sedentary and nonobese (BMI between 19.48 and $28.53 \mathrm{~kg} / \mathrm{m}^{2}$ ). Clinical characteristics and biological parameters were measured in fasting blood samples and presented in Table 1. Informed written consent was obtained from all participants before any aspect of the project was initiated.

\section{Exercise protocol}

The study workflow is illustrated in Figure 1. All subjects first completed a peak pulmonary oxygen uptake rate (maximal oxygen uptake $\left[\mathrm{VO}_{2} \max \right]$ ) test under fasting conditions, before and after the exercise training program. This test consisted of an incremental exercise to volitional fatigue on an electromagnetically braked cycle ergometer (Monark Ergomedic 839E, Vansbro, Sweden) during which pulmonary gas exchange was measured breath-by-breath with a gas analyzing system (Oxycon Pro Jaeger, Acertys, Aartselaar, Belgium). The participants then performed a 15 -min exercise bout, set at an intensity corresponding to $80 \%$ of the previously assessed $\mathrm{VO}_{2}$ max, and sWAT biopsies were collected under fasting conditions at rest (basal), $20 \mathrm{~min}\left(20^{\prime}\right), 1 \mathrm{~h}\left(60^{\prime}\right)$ and $4 \mathrm{~h}$ $\left(240^{\prime}\right)$ after cessation of exercise.

This entire procedure was repeated after the subjects had performed a 6-week intensive $\left(75-85 \% \mathrm{VO}_{2} \max \right)$ exercise program consisting of 45-min cycling sessions, 5 days per week. Tissue biopsies of the trained state were collected at least 5 days after the last training session in order to avoid any remaining single exercise bout effects. 


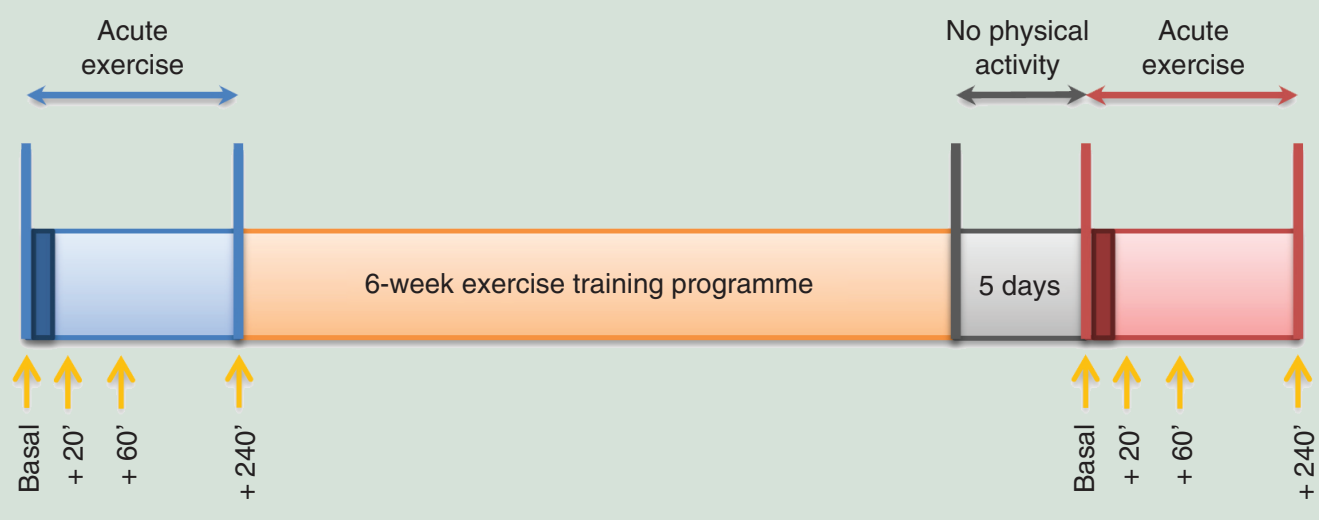

Figure 1. Workflow of the study. The 15 male volunteers first performed an intense $\left(80 \% \mathrm{VO}_{2} \max \right) 15$-min exercise bout on an electromagnetically-braked cycle ergometer. sWATs were collected under fasting conditions at rest (basal),

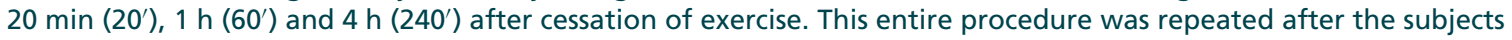
had performed a 6 -week intensive $\left(75-85 \% \mathrm{VO}_{2}\right.$ max) exercise program (45-min spinning classes, 5 days per week) at least 5 days after the last training session.

SWAT: Subcutaneous white adipose tissue; $\mathrm{VO}_{2}$ max: Maximal oxygen consumption.

All clinical investigations were conducted according to the principles expressed in the Declaration of Helsinki. The protocol of this study has been approved by the local Ethic Committee and the trial has been registered on ClinicalTrials.gov.

\section{Adipose tissue biopsies}

sWAT biopsies were obtained from periumbilical subcutaneous fat area as described [22], immediately snap-frozen and stored at $-80^{\circ} \mathrm{C}$ until further analysis.

\section{Nucleic acid purification}

Genomic DNA and total RNAs were purified from adipose tissue biopsies with the AllPrep DNA/RNA/miRNA Universal Kit (Qiagen, Hilden, Germany). The quality of recovered DNA and RNA was assessed by the 260/280 ratio, determined by spectrophotometry with a NanoDrop 2000 (Thermo Fisher Scientific, Roskilde, Denmark), as well as using the Agilent RNA 6000 Nano Kit (Agilent Technologies, Glostrup, Denmark) for RNA. DNA and RNA concentrations were determined with the Qubit dsDNA HS Assay Kit (Life Technologies, Roskilde, Denmark) and by spectrophotometry with a NanoDrop 2000, respectively. After purification, DNA and RNA samples were stored at $-80^{\circ} \mathrm{C}$ until further analysis.

\section{RNA sequencing}

Pre- and post-training RNA samples from eight participants were randomly chosen for transcriptomic sequencing. We analyzed only samples collected at basal and $240^{\prime}$ time points, as alterations in mRNA expression several-fold from basal levels are typically greatest at 3-12 h after cessation of exercise [23]. Total RNA (600 ng) was first depleted in rRNA depletion using the Ribo-Zero Magnetic Gold Kit (Human/Mouse/Rat; Epicentre, WI, USA) and rRNA-depleted samples were purified using Agencourt RNAClean XP (Beckman Coulter, CA, USA). Libraries were then prepared after rRNA depletion with the TruSeq Stranded Total Sample Prep Kit (Illumina, CA, USA). Last, libraries were quality-controlled using a Bioanalyzer instrument (Agilent Technologies) and subjected to 100-bp single-end sequencing on a HiSeq 2500 system (Illumina) at the Danish National High-Throughput DNA Sequencing Center. An average depth of 36.6 million reads was generated.

\section{Reduced representation bisulfite sequencing}

Pre- and post-training DNA samples from 12 and nine participants (nine paired samples), respectively, were randomly chosen for DNA CpG methylation analysis. Here, we analyzed samples collected at basal, 20', 60' and $240^{\prime}$ time points, as alterations in DNA methylation are very dynamic and can occur only 20 min after cessation of exercise [17]. RRBS library pool was prepared as previously described [24,25] and subjected to 50-bp single-end 
sequencing on a HiSeq 2500 system at The Danish National High-Throughput DNA Sequencing Center. On average, 15 million reads/sample were generated. Reads were trimmed for adapters and low-quality $3^{\prime}$ ends using the 'rrbs' setting of Trim Galore v.0.3.7 and Cutadapt v.1.4.2. Processed reads were mapped to the hg19 reference genome and methylation levels estimated using Bismark [26] and Bowtie2 [27]. Differential methylated regions between the respective time points $\left(20^{\prime}, 60^{\prime}\right.$ and $\left.240^{\prime}\right)$ and Basal were identified from the subset of CpG sites covered in at least $50 \%$ of the samples using BiSeq [28] with the model syntax y $\sim$ timepoint + subject.

\section{Bioinformatics}

RNA-seq analysis was performed as described [29]. Reads were processed with Trim Galore, mapped with the subread aligner and reads were assigned the ENSEMBLE genome using featureCounts [30], using standard settings. The samples had on average 25.3 million RNA reads assigned to genes, and the lowest and highest numbers were 21.6 and 29.6 million reads, respectively. Differential testing was performed using the edgeR function glmlRT [31], with a model of the form $\sim$ group + participant, where group encoded the four combinations of untrained/trained and Basal/240 min.

RRBS analysis was performed as described [32]. Reads were preprocessed with Trim Galore. Mapping and methylation level estimation were performed with Bismark [26], using standard settings. The average coverage was 8.8 million reads and varied from 2.7 to 17.5 million reads. Differential methylation was found using BiSeq [28], with a model formulated as $\sim$ timestamp + participant, where timestamp encoded the four time-point sequences. The trained and untrained states were analyzed separately.

In order to discriminate adipocyte-specific reads from those due to immune cell infiltration induced by acute exercise in our RRBS and RNA-seq datasets, we used available epigenetic and transcriptomic data profiling macrophages.

Macrophage methylation data (produced by the BLUEPRINT consortium, EGAD00001000914 and EGAD00001001139) were downloaded from http://dcc.blueprint-epigenome.eu/\#/datasets after data access agreement. Macrophage expression profiles were downloaded from Gene Expression Omnibus (accession number GSE36952). All datasets were processed using the same settings as the data generated in this study. Macrophage methylation percentages were calculated for the genomic clusters previously defined on white adipose tissue methylation data. For each participant, macrophage infiltration after training (20,60 and $240 \mathrm{~min}$ for methylation and 240 min for RNA) was calculated by minimizing:

$$
v_{t}-(1-x) v_{t_{\text {basal }}}+x v_{\text {macrophage }}
$$

subject to the constraint:

$$
0<x<1
$$

where $v_{t}, v_{\text {basal }}$ and $v_{\text {macrophage }}$ are the fractions of methylation or the $\log 2$ counts per million $(\log \mathrm{CPM})$ for expression at time point $t$, at the basal state or in macrophages, respectively and $x$ is the fraction of macrophage infiltration. Fraction of infiltration was calculated separately for the trained and the untrained state. The formula was solved using quadratic programming as implemented in the R package quadprog. Residual differences were calculated as:

$$
e=v_{t}-(1-x) v_{t_{\text {basal }}}+x v_{\text {macrophage }}
$$

For RNA-seq data, $e$ was calculated on $\log \mathrm{CPM}$, while for methylation data, CpGs were aggregated to an overall error of the cluster by taking the median. The distribution of residuals was observed to be symmetrically centered on zero. Individual clusters or genes whose change could be attributed to macrophage infiltration were identified by comparing them to the set of all clusters or genes using a Mann-Whitney $U$ test. 


\section{Principal component analysis}

Singular value decomposition based principal component analyses (PCAs) were conducted on all tested features in the RNA-seq and RRBS data, respectively. Data were projected onto the first and second principal components and visualized along with group-wise confidence intervals.

\section{Gene ontology term enrichment}

We used the Database for Annotation, Visualization and Integrated Discovery (DAVID) v6.8 [33,34] to investigate possible enrichment for gene ontology terms in the RNA-seq and RRBS datasets. For RRBS, we attributed each differentially methylated region (DMR) to the nearest gene based on the shortest distance to the transcription start site.

\section{Real-time quantitative PCR}

Pre- and post-training RNA samples from all participants were analyzed by quantitative PCR (qPCR) for expression of specific transcripts. Total RNA (300 ng) was used as a template for cDNA synthesis using the iScript cDNA Synthesis Kit (Bio-Rad, Copenhagen, Denmark). mRNA expression was then quantified from generated cDNA by real-time quantitative PCR, using Brilliant III Ultra-Fast SYBR Green QPCR Master Mix (Agilent Technologies), on a CFX96 Real-Time System Thermal Cycler (Bio-Rad). Primer sequences (Supplementary Table 1) were designed using Geneious computer software. qPCR data were analyzed according to the comparative cycle threshold method and were normalized by stable reference gene of GAPDH.

\section{Statistics}

Statistics were performed using GraphPad Prism software version 7 (GraphPad Software, Inc). For the analysis of qPCR data, we conducted a Student's t-test for paired samples on $\log 2$-transformed values. We considered that the difference between two conditions was statistically significant when ${ }^{*} \mathrm{p} \leq 0.05,{ }^{* *} \mathrm{p} \leq 0.01$ or ${ }^{\S} \mathrm{p} \leq 0.001$ and reported a tendency when $0.05<\mathrm{p}<0.1$.

\section{Results}

\section{Acute exercise, but not exercise training, changes gene expression in SWAT}

To determine the transcriptomic and the DNA methylation response of human adipose tissue to acute exercise in different states of exercise training, we collected sWAT from 15 men before and after a single exercise bout, which occurred before, and after a 6 -week endurance training program (Figure 1). We defined the intensity and duration of the acute exercises based on previous studies showing mRNA expression and DNA methylation changes in skeletal muscle $[17,35]$. The intensity and duration of the exercise training sessions were defined to obtain a post-training increase in $\mathrm{VO}_{2}$ max as reported [14,17]. Clinical characteristics and biological parameters are presented in Table 1. Expectedly, post-training values of $\mathrm{VO}_{2}$ max were increased by $15 \%$ compared with baseline (46.88 \pm 1.22 vs $54.97 \pm 1.17 \mathrm{ml} / \mathrm{min} / \mathrm{kg}, \mathrm{p}<0.001$; Table 1). Bodyweight was not changed after training but waist/hip ratio decreased $(0.92 \pm 0.01$ vs $0.88 \pm 0.01, \mathrm{p}<0.001)$, indicating abdominal fat loss (Table 1$)$.

We first performed a transcriptomic analysis by RNA-seq on sWAT collected before, and $240^{\prime}$ after each of the acute exercise bouts (Figure 1). The RNA-seq results were validated by RT-qPCR on 12 genes deliberately selected for having different expression patterns between basal and 240' (Supplementary Figure $1 \&$ Supplementary Table 2). PCA and hierarchical clustering of gene expression profiles for each individual sample at each time point revealed a gene expression shift after pre- and post-training acute exercise bouts (time point $240^{\prime}$ ), while basal profiles pre- versus post-training were similar (Figure 2A). This latter result was corroborated by differential expression analysis, which did not return any differentially expressed genes between the pre- and the post-training basal time points (Supplementary Table 3). In contrast, gene expression was readily changed in response to each acute exercise (Figure 2A). Interestingly, despite both single exercise bouts being performed at the same relative intensity, gene expression response was more pronounced in pretraining, compared with post-training condition. Among 16,242 total genes detected, $3882(23.9 \%)$ were differentially expressed after the acute exercise bout performed pretraining, while only 349 genes $(2.1 \%)$ were differentially expressed after the acute exercise bout performed post-training (Figure 2B \& Supplementary Tables $4 \& 5$ ). Among the differentially expressed genes, 2435 (62.7\%) were upregulated and 1447 (37.3\%) were downregulated after pretraining acute exercise, and 228 (65.3\%) were upregulated and 121 (34.7\%) were downregulated after post-training acute exercise (Figure 2C). Gene ontology enrichment analysis using the online tool DAVID revealed a strong enrichment for terms related to immune and 


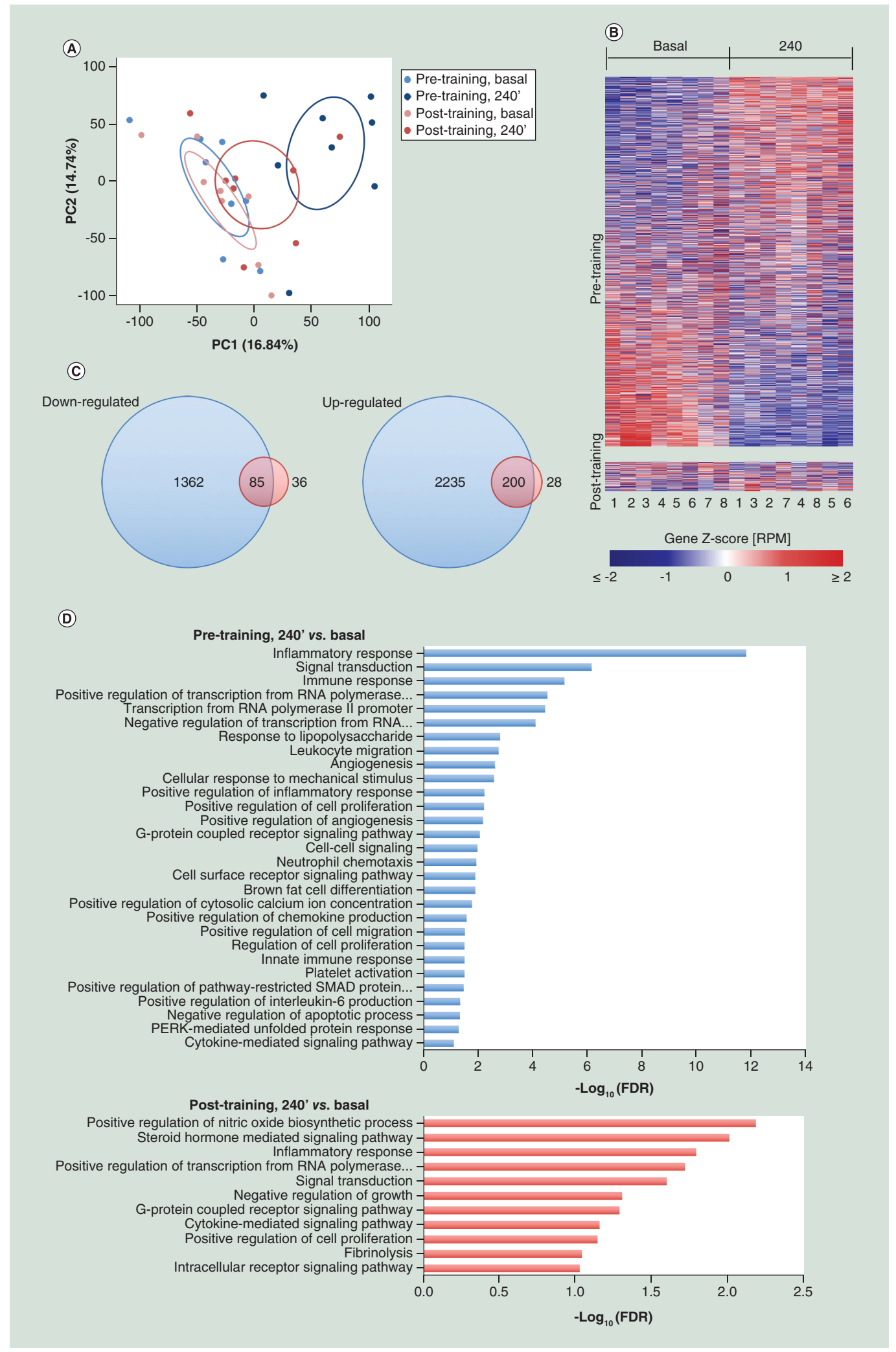

Figure 2. Transcriptomic changes induced by acute exercise in human subcutaneous white adipose tissue (RNA sequencing). (A) PCA of RNA-seq data for the major two PCs. The 95\% confidence ellipses are shown for each group. (B) Heatmaps of the differentially expressed genes in adipose tissue before (basal) and after $\left(240^{\prime}\right)$ the acute exercise bout, before (pretraining) or after (post-training) the 6-week exercise training program. FDR $<0.1$. (C) Venn diagram representing the number of genes that were upregulated or downregulated after the acute exercise bout, before (pre-training) and after (post-training) the 6-week exercise training program. The intersection represents genes that were regulated in both conditions. FDR $<0.1$. (D) Gene ontology analysis (biological process) of differentially expressed genes after acute exercise, before (pre-training) and after (post-training) the exercise training intervention. FDR $<0.1$.

FDR: False discovery rate; PC: Principal component; PCA: Principal component analysis; RNA-seq: RNA sequencing; sWAT: Subcutaneous white adipose tissue. 


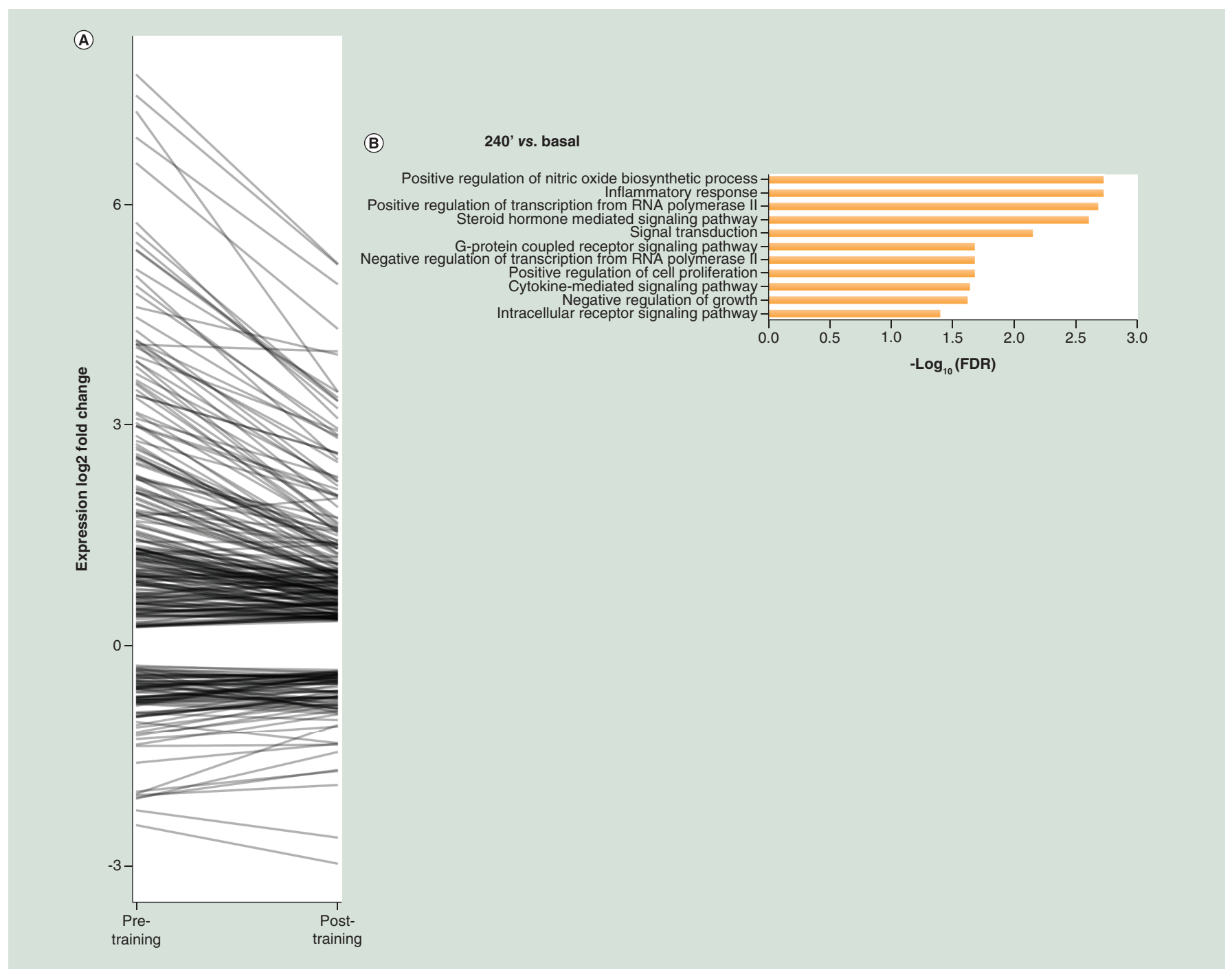

Figure 3. Modulation of the immune/inflammatory response induced by acute exercise with exercise training. (A) Representation of the variation in the fold change of expression induced by acute exercise of the 285 genes differentially expressed in both pre- and post-training conditions between these two time points. FDR $<0.1$. (B) Gene ontology analysis (biological process) of the 285 genes that were differentially expressed after acute exercise in both pre- and post-training conditions. FDR $<0.1$. FDR: False discovery rate.

inflammatory response, in both pre- and post-training datasets, but with a higher enrichment score at pretraining (Figure 2D). Among all differentially expressed genes, 285 genes (corresponding to $7.3 \%$ of genes differentially expressed pretraining and $81.7 \%$ of genes differentially expressed post-training) were regulated by acute exercise in both pre- and post-training conditions. Expression fold change for these genes was globally lower post-training ( 2.45 vs 6.86; $\mathrm{p}<0.001)$ (Figure $3 \mathrm{~A})$. Similar to the list represented by the sum of all the differently expressed genes, genes in common between pre- and post-training datasets showed main gene term ontologies related to inflammatory response (Figure 3B). Thus, our results show that most exercise-responsive genes in adipose tissue are related to inflammatory response and that the untrained state is associated with a higher magnitude in gene expression response to acute exercise.

\section{Monocytes or macrophages infiltrate sWAT after acute exercise}

Adipose tissue is constituted of a variety of nonadipocyte cells such as stromal vascular cells and immune cells, which participate in the adipose tissue homeostasis and function [36,37]. Given that exercise affects chemotaxis and infiltration of immune cells [38] and that we found an enrichment in genes related to immune and inflammatory 
(A)
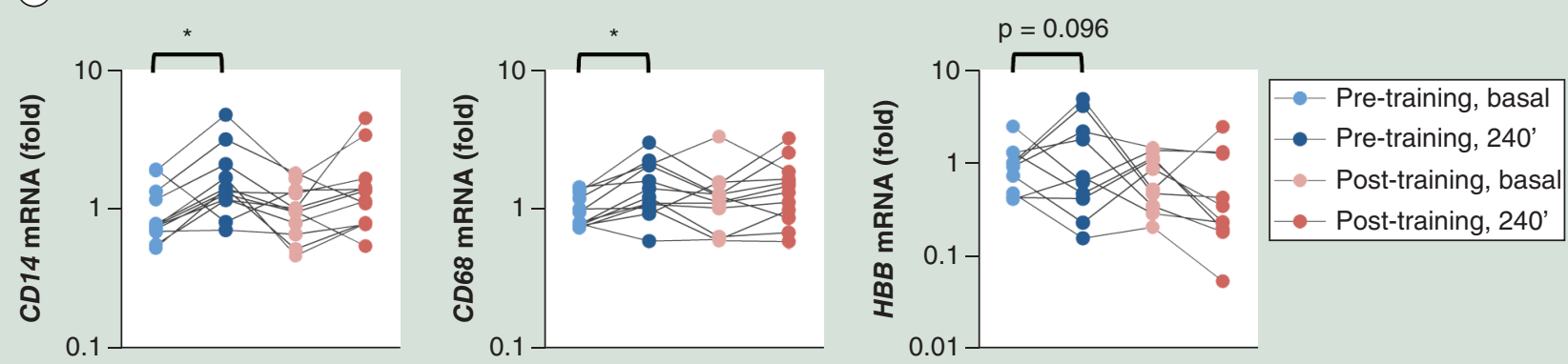

(B)

Pre-training, 240' vs. basal

Post-training, 240 ' vs. basal
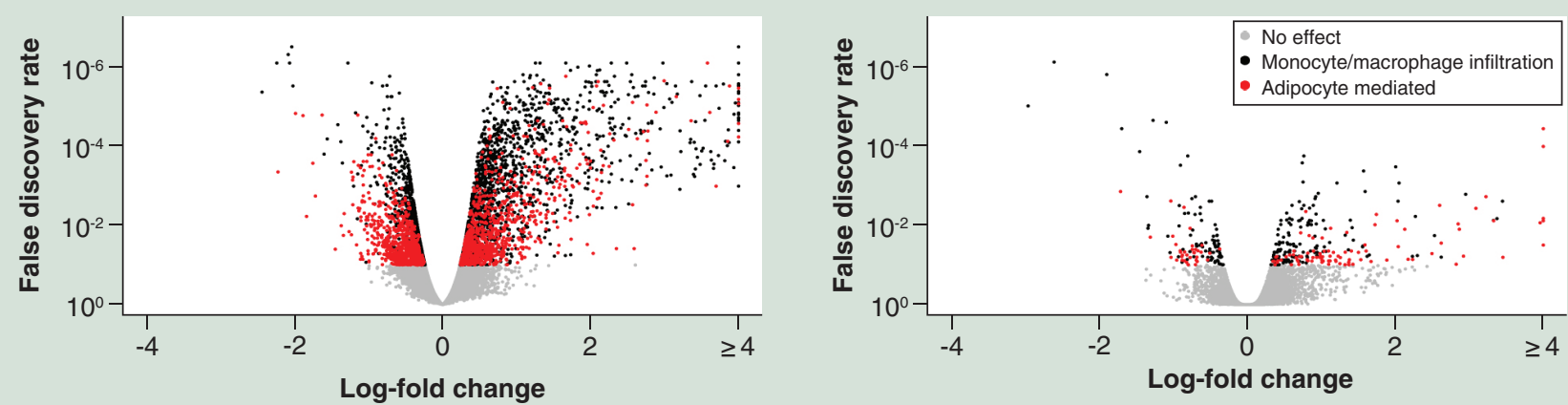

(C)

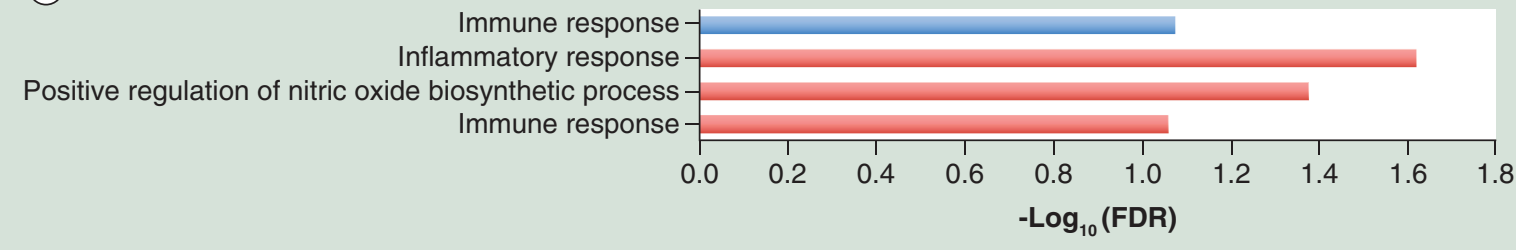

Figure 4. Evaluation of monocyte/macrophage infiltration induced by acute exercise and its impact on RNA sequencing data.

(A) RT-qPCR analysis of CD14 $(n=12), C D 68(n=12)$ and HBB $(n=10)$ mRNA expression in adipose tissue biopsies collected at basal time (basal) and $4 \mathrm{~h}\left(240^{\prime}\right)$ after acute exercise, before (pre-training) and after (post-training) the exercise training program. ${ }^{*} \mathrm{p}=0.021$ for $C D 14$ and ${ }^{*} \mathrm{p}=0.011$ for $C D 68240^{\prime}$ versus basal, pretraining. All graphs represent $2^{-\Delta \mathrm{Ct}}$ values expressed relatively to the mean of pretraining $2^{-\Delta C t}$ values obtained for all subjects, which was set at 1 . Vertical axis scale is logarithmic base 10. (B) Volcano plots showing directional changes of the differentially expressed genes in adipose tissue after the acute exercise bout, before (pre-training) or after (post-training) the 6-week exercise training program. The genes differentially expressed due to immune cell infiltration (black dots) and those related to changes in adipocyte functions (red dots) are shown. FDR $<0.1$. (C) Gene ontology analysis (Biological Process) of the adipocyte-related genes that were differentially expressed after acute exercise in both pre- (blue) and post-training (red) conditions. FDR $<0.1$.

FDR: False discovery rate; qPCR: Quantitative PCR; RNA-seq: RNA sequencing.

response after acute exercise, we investigated whether infiltration of immune cells after acute exercise could cause an increased detection of inflammatory response genes. Thus, we analyzed the expression of immune cell surface markers in our RNA-seq dataset. We found CD3G (T-cell marker), CD19 and MS4A1 (B-cell markers) were not detected while $C D 4$ and $C D 8 A$ genes (T-cell markers) were detected but not differentially expressed after acute exercise (Supplementary Tables $4 \& 5$ ). On the other hand, the monocyte/macrophage marker CD14 was upregulated by pretraining acute exercise $(\mathrm{FC}=2.07, \mathrm{p}=3.18 \mathrm{E}-06$; Supplementary Tables $3 \& 4)$, a result that was confirmed by RT-qPCR (Fold Change $(\mathrm{FC})=1.76, \mathrm{p}=0.021$ ) (Figure $4 \mathrm{~A}$ ). In addition, $C D 68$, another macrophage marker, was also upregulated by pretraining acute exercise $(\mathrm{FC}=1.54, \mathrm{p}=0.011$ ) (Figure $4 \mathrm{~A})$. Expression of $H B B$, used here as a marker of total blood contamination of the adipose tissue biopsy like previously described [8], was unchanged after acute exercise (Figure 4A \& Supplementary Tables $4 \& 5$ ). Thus, our results suggest that 
only specific immune cells infiltrate adipose tissue after acute exercise. Nevertheless, our results indicate that our transcriptomic results could be biased by infiltration of immune cells postexercise.

In an attempt to quantify the impact of immune cell infiltration on our transcriptomic results and to identify adipocyte-specific response, we subtracted immune cell specific genes from our transcriptomic dataset (Figure 4B \& Supplementary Tables 4 \& 5). After removal of genes more expressed in immune cells than in adipocytes, we only found 1586 differentially expressed genes regulated by acute exercise pretraining (i.e., $40.9 \%$ of the original dataset) and 127 post-training (36.4\%). Strikingly, even though we stringently removed genes related to immune cells from the original list of differentially expressed genes, gene ontology enrichment analysis still showed enrichment in inflammatory responses. Thus, the transcriptomic response of adipose tissue to acute exercise may be driven by both infiltration of immune cells and a specific transcriptomic response in adipocyte.

\section{Acute exercise \& exercise training remodel DNA methylation in human sWAT}

We next performed genome-wide DNA methylation of adipose tissue DNA in all time points using RRBS, a bisulfite sequencing technique allowing to enrich for CG dinucleotides (Supplementary Tables 6-8). As expected, given the high CG representation in gene promoters, we found most of DMRs located in gene promoters (Figure 5A). Similar representation of highly- or lowly methylated sequences across samples validated our RRBS analysis (Figure 5B). Analysis of basal states returned 240 DMRs regulated by exercise training, which corresponds to 218 unique gene IDs, with 130 hypomethylated DMRs and 110 hypermethylated (Supplementary Table 8). Using PCA and hierarchical clustering analysis of the genome-wide DNA methylation profiles, we found a marked shift after the acute exercise bout (time point 240'), in pretraining condition only (Figure 5C). Analysis of DNA methylation response to each acute exercise bout returned 662 and 888 DMRs, corresponding to 580 and 770 unique gene IDs, regulated by the acute exercise performed pre- and post-training, respectively (Supplementary Tables $7 \& 8$ ). Among these DMRs, DNA methylation level was lower for 387 and 564 and higher for 275 and 324 in response to acute exercise, with absolute changes ranging from $<0.001$ to 76.6 and $99.8 \%$ for pre- and post-training, respectively. These results are in striking contrast with our transcriptomic analysis, where higher magnitude in response was found before training. Among all genes located at proximity of the regions differentially methylated after acute exercise, only 32 were found both in pre- and in post-training datasets. Gene ontology analyses of genes located at proximity of all DMRs did not return any enrichment using DAVID with FDR $\geq 0.5$. Thus, our results do not support a role of DNA methylation in a unified, functional gene expression response.

For DNA methylation results also, we evaluated monocyte/macrophage infiltration by measuring CD14 mRNA at the 20-, 60- and 240-min time points after acute exercise (Figure 6A), and estimated its impact on RRBS data by calculating the fraction of infiltration (Figure 6B) and identifying immune cell-related DMRs (Figure 6C \& Supplementary Tables $6 \& 7$ ). After removal of immune cell-specific methylated regions, a majority of DMRs were left; 503 DMRs were left after acute exercise, pretraining (i.e., $76.0 \%$ of the original dataset) and 703 post-training (79.2\%). The remaining DMRs were associated to 445 and 639 unique gene IDs, respectively. Thus, our results further reinforce that immune cell infiltration after exercise modestly affected our analyses and show that both acute and chronic exercise induce DNA methylation changes in adipose tissue.

\section{DNA methylation changes are partly linked to gene expression response to exercise training}

In order to determine the biological relevance of exercise-induced DNA methylation changes in sWAT, we applied a threshold of $>5 \%$ for absolute difference in methylation to RRBS datasets. This resulted in 219 and 359 remaining DMRs, corresponding to 191 and 335 unique gene IDs, regulated by acute exercise performed preand post-training, respectively. Among these DMRs, DNA methylation level was lower for 107 and 239 DMRs and higher for 112 and 120 DMRs in response to acute exercise, for pre- and post-training, respectively. Only 33 remained after restriction of the list to DMRs regulated by exercise training, all located at proximity of a gene and mostly hypomethylated. By crossing RRBS ( $\mathrm{q}<0.1$ and absolute difference in methylation $>5 \%$ ) and RNA-seq ( $<<0.01$ and absolute $\log \mathrm{FC}<0.5)$ datasets, we identified 32 (pretraining) and six (post-training) overlapping genes (Table 2), notably including $A D R A 2 A, F O S L 1, M E T R N L$ and $R A R A$, well known for their role in adipocyte differentiation and metabolism, but also CBLB, GPR132 and RELT, involved in various immune functions. A Spearman's correlation analysis pointed at a modest association between DNA methylation and gene expression at genes near DMRs $(R=0.16 ; p=0.32$ ) (Supplementary Figure 3$)$. Our data suggest that gene expression is only partly regulated by DNA methylation in human adipose tissue. 
Table 2. List of genes with absolute difference in methylation $<5 \%(q<0.1)$ in reduced representation bisulfite sequencing dataset and absolute logFC $<0.5$ in RNA sequencing dataset $(q<0.1)$, comparing adipose tissue biopsies collected at basal time and $20 \mathrm{~min}\left(20^{\prime}\right), 1 \mathrm{~h}\left(60^{\prime}\right)$ or $4 \mathrm{~h}\left(240^{\prime}\right)$ after acute exercise, before (pre) or after (post) the exercise training program.

\begin{tabular}{|c|c|c|c|c|c|}
\hline Time point & Gene & DMR location & $\begin{array}{l}\text { Differential } \\
\text { methylation (\%) }\end{array}$ & Gene feature & logFC RNA-seq \\
\hline Pre-20' & $A D R A 2 A$ & chr10:112838452-112838500 & 40.90 & Exon 1 of 1 & 0.78 \\
\hline Pre-20' & $A L X 4$ & chr11:44325828-44325850 & -16.72 & Intron 3 of 3 & -1.20 \\
\hline Pre-20' & $A L X 4$ & chr11:44326173-44326177 & -8.86 & Intron 3 of 3 & -1.20 \\
\hline Pre-20' & $A P O B R$ & chr16:28509350-28509350 & 20.05 & Intron 2 of 3 & 2.79 \\
\hline Pre-20' & ARHGAP39 & chr8:145806295-145806394 & -6.28 & Exon 4 of 13 & 0.91 \\
\hline Post- $240^{\prime}$ & $C B L B$ & chr3:105587187-105587227 & -70.43 & Promoter & 0.51 \\
\hline Pre-20' & $D D \times 39 A$ & chr19:14519683-14519716 & 18.71 & $3^{\prime}$ UTR & 0.88 \\
\hline Pre-60' & FOSL1 & chr11:65667275-65667275 & 7.25 & Promoter & 7.76 \\
\hline Pre-20' & GLIS3 & chr9:4299330-4299331 & -12.51 & Promoter & 0.54 \\
\hline Pre-20' & GNA15 & chr19:3116626-3116626 & 8.24 & Intron 4 of 6 & 0.68 \\
\hline Pre-20' & GNA15 & chr19:3136424-3136424 & -25.40 & Promoter & 0.68 \\
\hline Pre-20' & GNA15 & chr19:3136658-3136671 & -38.51 & Promoter & 0.68 \\
\hline Pre-240' & GPR132 & chr14:105554651-105554651 & 11.25 & Distal intergenic & 0.74 \\
\hline Pre-20' & HELZ2 & chr20:62198960-62198984 & -45.75 & Promoter & 1.08 \\
\hline Pre-20' & $H L X$ & chr1:221060851-221060879 & -27.64 & Downstream 2-3 kb & 1.09 \\
\hline Pre-60' & HNRNPF & chr10:43891725-43891725 & -6.11 & Promoter & 0.62 \\
\hline Pre-20' & HSD17B14 & chr19:49340623-49340712 & -5.20 & Promoter & 0.66 \\
\hline Pre-20' & $\operatorname{IRX} 1$ & chr5:3600863-3600896 & -13.14 & Intron 3 of 3 & -0.73 \\
\hline Pre-20' & METRNL & chr17:81050773-81050773 & 37.70 & Promoter & 0.64 \\
\hline Pre-20' & MMP25 & chr16: 3096513-3096515 & -8.76 & Promoter & 3.96 \\
\hline Pre-240' & MYADM & chr19:54372662-54372713 & 11.12 & Promoter & 1.08 \\
\hline Post-240' & $N G F R$ & chr17:47572710-47572715 & -10.63 & Promoter & 1.39 \\
\hline Pre-240' & $P 2 R X 1$ & chr17:3819131-3819131 & -15.87 & Promoter & 1.99 \\
\hline Pre-240' & $P 2 R \times 1$ & chr17:3819419-3819419 & -36.63 & Promoter & 1.99 \\
\hline Post- $60^{\prime}$ & PITPNC1 & chr17:65375129-65375131 & 8.93 & Intron 1 of 9 & 0.74 \\
\hline Pre-240' & RARA & chr17:38473194-38473222 & 29.95 & Intron 1 of 8 & 1.32 \\
\hline Pre-20' & RASGEF1B & chr4:82393130-82393130 & 7.24 & Promoter & 0.99 \\
\hline Pre-20 & $R E L T$ & chr11:73054326-73054326 & 42.48 & Intron 1 of 20 & 2.18 \\
\hline Pre-20' & RNF217 & chr6:125283783-125283783 & -13.66 & Promoter & 0.84 \\
\hline Pre-20' & S1PR4 & chr19:3179742-3179742 & -58.10 & Exon 1 of 1 & 1.24 \\
\hline Post-20' & SERTAD1 & chr19:40931833-40931836 & -14.43 & Promoter & 1.01 \\
\hline Pre-20' & SIK1 & chr21:44819040-44819040 & -35.36 & Distal intergenic & 0.91 \\
\hline Post- $60^{\prime}$ & SIX4 & chr14:61190530-61190607 & -41.49 & Promoter & -0.72 \\
\hline Pre-240' & $S L C 2 A 14$ & chr12:8025452-8025461 & -5.27 & Promoter & 2.72 \\
\hline Pre-240' & $S L C 45 A 4$ & chr8:142228845-142228845 & 10.25 & Exon 4 of 7 & 0.63 \\
\hline Pre-240' & SPTBN5 & chr15:42174496-42174505 & -21.75 & Exon 11 of 68 & 0.90 \\
\hline Pre-240 & SYTL1 & chr1:27676256-27676310 & 11.18 & Exon 8 of 15 & 1.59 \\
\hline Pre-20 & $T B X 4$ & chr17:59532043-59532043 & -5.90 & Intron 1 of 8 & -0.54 \\
\hline Post-240' & TGFA & chr2:70780866-70780888 & -8.31 & Promoter & 1.32 \\
\hline Pre-20' & TMEM204 & chr16:1574847-1574874 & 5.84 & Exon 14 of 22 & -0.59 \\
\hline Pre-60' & ZSWIM4 & chr19:13919887-13919905 & 6.87 & Exon 5 of 13 & 1.07 \\
\hline
\end{tabular}




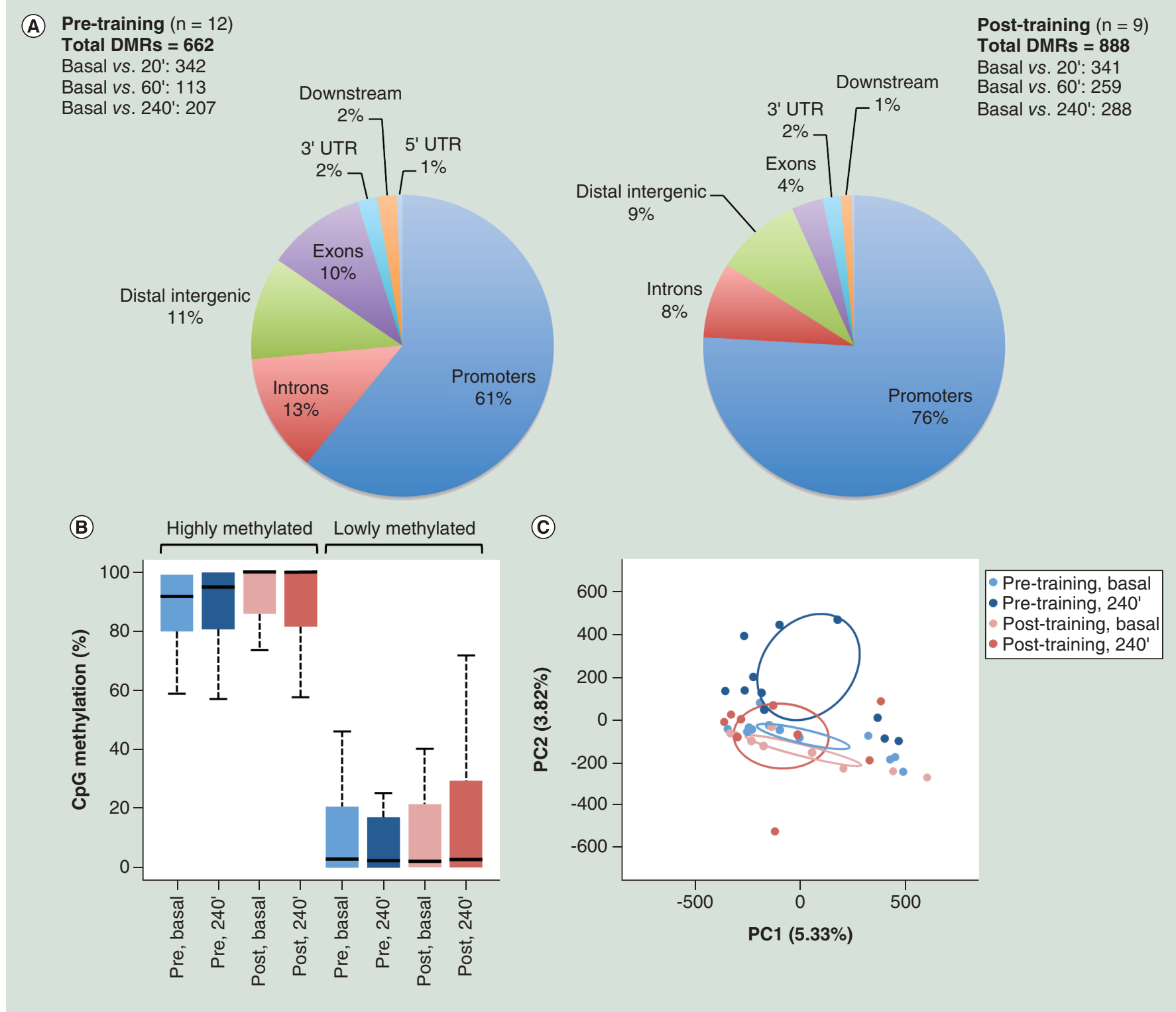

Figure 5. DNA methylation changes induced by acute exercise in human subcutaneous white adipose tissue reduced representation bisulfite sequencing (rrbs). (A) DMR location in relation to the nearest gene. (B) Validation of RRBS results by visualization of the representation of highly- and lowly-methylated sequences (information obtained from the Epigenome Roadmap) across samples. (C) PCA of RRBS data for the major two PCs. The $95 \%$ confidence ellipses are shown for each group.

DMR: Differentially methylated region; PC: Principal component; PCA: Principal component analysis; RRBS: Reduced representation bisulfite sequencing; sWAT: Subcutaneous white adipose tissue.

\section{Discussion}

This study provides a detailed map of transcriptomic and DNA methylation changes induced by acute exercise and exercise training in human sWAT. We show that genes related to inflammation are differentially expressed after acute exercise, with a higher magnitude at the untrained, compared with the trained state. Our computational analyses ruled out a bias for exercise-induced immune cell infiltration in our transcriptomic results. We found that only the trained state was associated with an overlap between gene expression and DNA methylation changes. Our results suggest that, in adipose tissue, DNA methylation remodeling is involved in the long-term, rather than short-term transcriptomic adaptation.

The recruitment of immune cells, essentially macrophages, in adipose tissue is an important feature of obesityinduced inflammation and is implicated in the development of metabolic diseases [39,40]. Although production and 


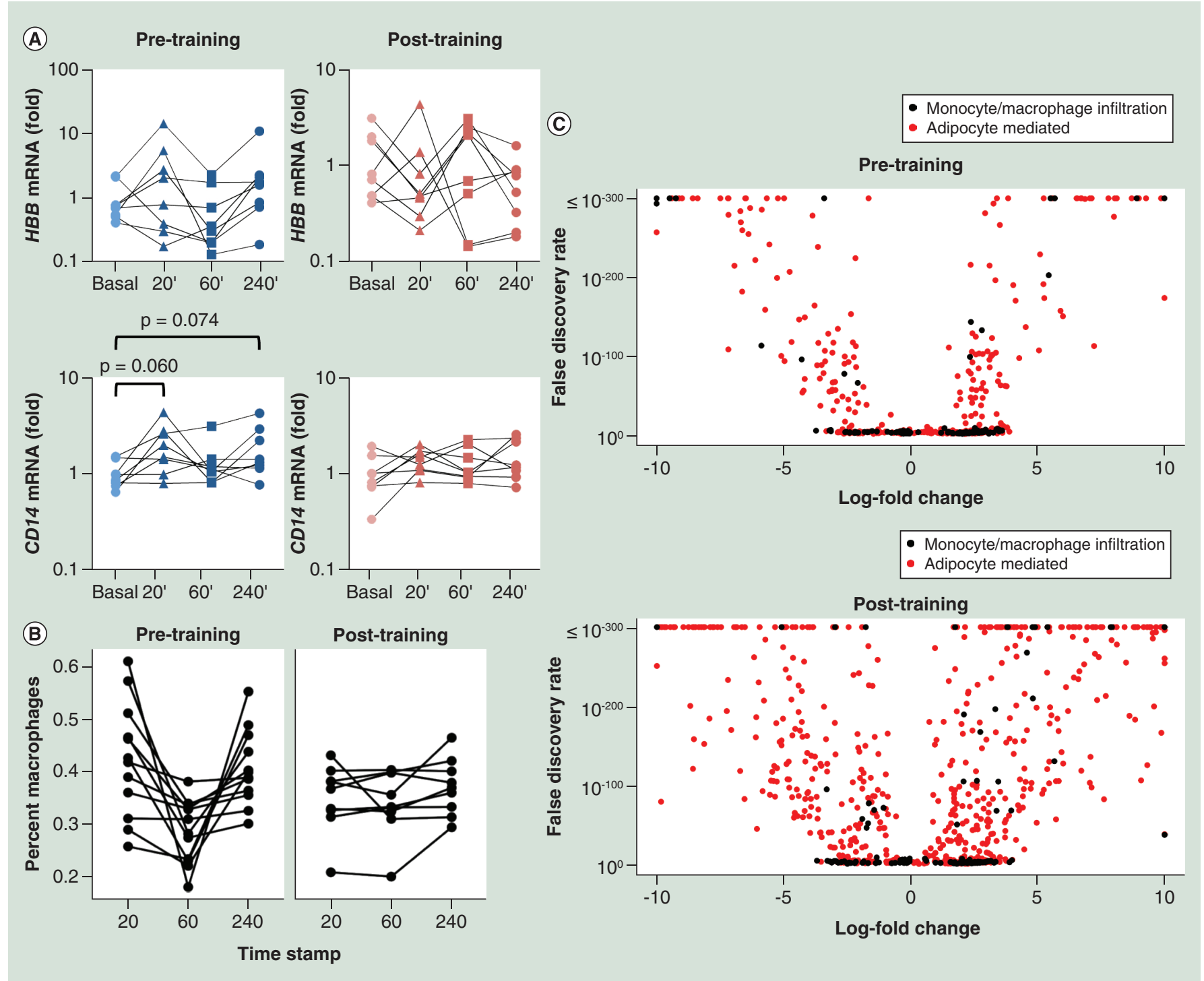

Figure 6. Evaluation of monocyte/macrophage infiltration induced by acute exercise and its impact on reduced representation bisulfite sequencing data. (A) qPCR analysis of $H B B(n=8)$ and CD14 $(n=8)$ mRNA expression in adipose tissue biopsies collected at basal time (basal), $20 \mathrm{~min}\left(20^{\prime}\right), 1 \mathrm{~h}\left(60^{\prime}\right)$ and $4 \mathrm{~h}\left(240^{\prime}\right)$ after acute exercise, before (pretraining) or after (post-training) the exercise training program. All graphs represent $2^{-\Delta C t}$ values expressed relatively to the mean of pretraining $2^{-\Delta C t}$ values obtained for all subjects, which was set at 1. Vertical axis scale is logarithmic base 10. (B) Estimated macrophage infiltration at 20,60 and 240 min after an acute bout of exercise, before (pretraining) or after (post-training) the exercise training program. A fraction of 0 would represent tissue no more infiltrated than at rest, while a fraction of 1 would be a pure macrophage sample. Connected point belong to the same participant. (C) Volcano plots showing directional changes of the DMRs in adipose tissue after the acute exercise bout, before (pretraining) or after (post-training) the 6-week exercise training program. The regions differentially methylated due to immune cell infiltration (black dots) and those related to changes in adipocyte functions (red dots) are shown. FDR $<0.1$.

DMR: Differentially methylated region; qPCR: Quantitative PCR; RRBS: Reduced representation bisulfite sequencing.

release of inflammatory cytokines and chemokines (also called adipocytokines) by adipose tissue macrophages has been mainly reported in obesity [39,41,42], other stressors such as exercise might represent a stress signal leading to the modulation of monocyte chemotaxis, differentiation into macrophages and activation. It is well documented that acute exercise increases expression and circulating levels of many inflammatory molecules such as cytokines and chemokines [43-51]. While many studies focused on skeletal muscle, only a few have investigated adipose tissue secretion following an acute exercise in nonobese individuals [43,46-51]. These studies notably report changes in $L E P, A D I P O Q, T N F$ and $N A M P T$ mRNA expression just after the exercise session and/or during the following hours period. Our RNA-seq data clearly show that the whole adipose tissue is highly stimulated by a single 
acute exercise session and suggest modulation of the inflammatory response through secretion of proinflammatory molecules into the bloodstream. While our results could be caused by exercise-induced macrophage infiltration and not activation of inflammation-related genes in adipocytes, our bioinformatics analyses ultimately demonstrated that adipocytes readily respond to exercise by modulating transcriptomic response of proinflammatory genes. Of note, given that gene expression has been analyzed from sWAT biopsies collected $4 \mathrm{~h}$ after the end of the acute exercise bout and, given that during this time three other adipose tissue biopsies have been taken, we cannot exclude that the genomic response we observe is not caused, at least in part, by an acute inflammatory response to previous biopsy collection.

Our study shows that gene expression response to acute exercise is more pronounced at the untrained, compared with the trained state. In other words, gene expression is more pronounced before the participants have performed a 6-week endurance training program. It is noteworthy that both acute exercise bouts (pre- and post-training) were performed at the same relative intensities and the same duration, which implicates that the higher magnitude in inflammation-related gene response is not caused by exercise intensity or duration. Yet, factors such as catecholamine could be responsible for the difference in gene expression magnitude between the untrained and the trained state, since postexercise plasma adrenaline concentration is significantly higher in endurance-trained than in untrained subjects, even when working at the same relative intensity [52].

In vivo measurements in a range of different subjects and exercise protocols using microdialysis and arteriovenous difference provided direct evidence that fatty acids are mostly mobilized from sWAT during low- to moderateintensity exercise, with only a small contribution coming from visceral fat. However, direct measurements in abdominal sWAT have shown that fatty acid mobilization from this fat depot does not rise proportionally to exercise intensity, indicating that alternative fat stores such as intramuscular fat are used when exercise intensity increases [8]. As intramuscular fat tends to be greater in trained individuals, and therefore contribute significantly to whole-body fat oxidation, this could partly explain the lower magnitude in adipose tissue transcriptional response we observed after training.

Possibly, inflammation-related genes become transcriptionally resistant after training. Such dampening of the transcriptomic response is consistent with the decrease in the adaptive response across repeated training sessions [53]. We can only speculate that expression of genes related to inflammation is particularly important in the early phase of training adaptation and participates in early structural changes in adipose tissue. In this hypothetical model, once structural changes are established, a chronic transcriptional activation of inflammatory genes would not be needed. Consistent with such hypothesis, eccentric exercise-induced muscle damage is associated with elevated markers of neutrophil activation in untrained, but not well-trained men, despite similar magnitude in muscle tissue damage $[54,55]$. Here, we did not find any association between DNA methylation and gene expression changes after acute exercise, suggesting the dampening of the transcriptomic response to acute exercise is unlikely to be carried by DNA methylation. The pathways by which the inflammatory response is attenuated are yet to be identified. Specific exercise-induced intracellular pathways that are critical to the inflammatory response may be dampened after training while other pathways remain identically activated at the same relative intensity. Postexercise production of reactive oxygen species (ROS), represents a likely candidate pathway as postexercise ROS production is lower in trained individuals [56] and given the documented role of ROS production in tissue inflammation [57].

The beneficial effect of exercise training on adipose tissue metabolism is ultimately the result of sustained changes induced by repeated transient acute exercise bouts, as already demonstrated in skeletal muscle [58]. Contradictory to previous studies realized by Rönn et al. [5,14], we did not find any change in basal gene expression between pre- and post-training transcriptomic profiles. This discrepancy in the results could be due to the different techniques used for mRNA expression profiling (RNA-seq vs array) or the difference in the exercise training protocol, such as the length of the intervention (3 vs 6 months). Similarly, we found only 20 genes differentially methylated after exercise training in common with Rönn et al., while 7663 genes were reported [14]. Again, these conflicting results could be explained by the difference in the techniques used for DNA methylation profiling (RRBS vs array), in other words, the Infinium Human Methylation 450 Bead Chip, which probed 485,577 CpG sites, and RRBS, which interrogates approximately 10 million CpGs sites. The discrepancy in DNA methylation results could therefore be caused by the different genomic positions of the CpGs covered in these two separate assays.

In the present study, we report acute changes in DNA methylation in sWAT, which were induced by only one session of high-intensity exercise. Based on gene ontology analyses, we found no association between exerciseinduced alterations in DNA methylation and gene expression, suggesting that DNA methylation does not drive the transcriptional response of genes related to inflammation in sWAT. A possible explanation for the relative lack 
of association between DNA methylation and mRNA expression could lay on the time course of sample collection. Given the highly dynamic nature of demethylation-remethylation process and its variability in time for each gene $[17,18]$, even though we profiled DNA methylome at 20-min, 1-h and 4-h recovery after the acute exercise bout, we analyzed mRNA expression in biopsies collected at 4-h recovery only. Therefore, we could have missed the time point $(s)$ for which epigenetic-regulated genes would have shown a concordant change in transcriptional activation and DNA methylation profile in response to acute exercise. Acute exercise remodels DNA methylation in skeletal muscle cell and tissue, which suggested a role of DNA methylation in exercise-induced gene expression [17,18,59]. It is noteworthy that in the latter studies, whole-genome DNA methylation and transcriptomic analysis were not systematically performed. Instead, specific candidate genes known to be transcriptionally upregulated by exercise were studied. Thus, it is possible that a causal relationship between DNA methylation and gene expression after acute exercise readily exists in white adipose tissue, but only for a small subset of genes. Of potential interest, in the present study, we identified some gene-specific alterations that might be relevant for their role in adipogenesis, fat browning or immunity. The $A D R A 2 A$ gene encodes for a G-protein-coupled receptor regulating neurotransmitter release from sympathetic nerves and from adrenergic neurons. Its activation by catecholamines is notably involved in the control of white preadipocyte proliferation [60] and leads to reduced lipolysis in adipocytes. Exercise training reduces $\alpha(2)$-adrenergic antilipolytic effect in human sWAT, therefore improving lipid mobilization and lipid oxidation [61,62]. FOSL1 is a component of the AP-1 transcriptional complex which has been shown to suppress adipogenesis in favor of osteogenic cell fate. Mice overexpressing fos $l 1$ developed progressive osteosclerosis as a result of accelerated osteoblast differentiation, and also a severe general lipodystrophy [63]. METRNL is a circulating factor that is shown to be highly expressed in white adipose tissue and to beneficially affect energy metabolism in mice. Indeed, elevated circulating levels of METRNL increases energy expenditure and improves glucose tolerance and the expression of genes associated with beige fat thermogenesis and anti-inflammatory cytokines [64]. In adipose tissue, METRNL promotes white adipocyte differentiation, expandability and lipid metabolism, and inhibits adipose inflammation to form functional fat, which contributes to its activity against insulin resistance [65]. RARA is a nuclear retinoic acid receptor which is mostly metabolized and stored in the liver and in adipose tissue. By binding to its receptor, the retinoic acid plays a crucial role in the signaling pathway of many other factors and particularly in the fatty acid signaling pathway. Many in vitro studies have shown different actions of retinoic acid in the control of adipose tissue proliferation and differentiation [66]. Of interest, obesity is associated with lower level of RARA expression in human subcutaneous adipose tissue [67]. CBLB is an E3 ubiquitin ligase that has been identified as a negative regulator of macrophage migration and activation in adipose tissue and associated with insulin resistance in obesity [68]. GPR132 is a stress-inducible receptor which mainly regulates chemotaxis and other immune functions of lymphocytes and macrophages. It is notably a receptor for oxidized fatty acids, which may be a sensor of lipid overload and oxidative stress, and which has a protective role against atherosclerosis [69]. RELT is a member of the TNF-receptor superfamily and has been shown to activate the NF- $\kappa$ B pathway and stimulate T-cell proliferation in the presence of $\mathrm{CD} 3$ signaling, which suggests its regulatory role in immune response [70]. Thus, our results, by highlighting several genes controlling adipose tissue function, indicate that training-induced DNA methylation may modulate adipocyte function after exercise.

\section{Conclusion}

In summary, acute exercise causes a prominent reprogramming of both transcriptome and epigenome in sWAT and adipocyte, notably affecting immune and inflammatory pathways. With regular exercise training, this response markedly diminishes, suggesting the establishment of downregulation mechanisms. Long-term regulation of gene expression at the trained state might be governed, at least in part, by DNA methylation. Experiments using nuclease dead Cas9 system fused to DNA-modifying enzymes and targeting specific exercise-responsive genes in adipose tissue should be performed to determine the definitive causal relationship between DNA methylation and gene expression in adipose tissue after exercise. While DNA methylation may partly control gene expression after exercise in adipose tissue, the physiological relevance of DNA methylation changes that are not associated with gene expression changes remains to be established. 
Summary points

- Acute exercise causes subcutaneous white adipose tissue (sWAT) infiltration with activated macrophages.

- The sWAT transcriptome after acute exercise is mainly enriched for genes involved in inflammation.

- Endurance exercise training mitigates sWAT transcriptomic response induced by acute exercise.

- Endurance training modifies the transcriptomic signature of adipose tissue.

- Acute exercise induces a very dynamic DNA methylation-remethylation process in sWAT.

- Exercise training remodels sWAT DNA methylation profile.

- Few genes involved in adipogenesis and immunity are affected both at the transcriptomic and epigenetic level at the trained state.

Financial \& competing interests disclosure

This study makes use of data generated by the BLUEPRINT Consortium. A full list of the investigators who contributed to the generation of the data is available from www.blueprint-epigenome.eu. Funding for the project was provided by the European Union's Seventh Framework Programme (FP7/2007-2013) under grant agreement no 282510 - BLUEPRINT. The authors have no other relevant affiliations or financial involvement with any organization or entity with a financial interest in or financial conflict with the subject matter or materials discussed in the manuscript apart from those disclosed.

No writing assistance was utilized in the production of this manuscript.

\section{Ethical conduct of research}

All sample collections have been conducted under local ethical regulation and approval.

\section{Open access}

This work is licensed under the Attribution-NonCommercial-NoDerivatives 4.0 Unported License. To view a copy of this license, visit http://creativecommons.org/licenses/by-nc-nd/4.0/

\section{References}

Papers of special note have been highlighted as: $\bullet$ of interest; $\bullet \bullet$ of considerable interest

1. Frayn KN. Fat as a fuel: emerging understanding of the adipose tissue-skeletal muscle axis. Acta Physiol. 199(4), 509-518 (2010).

2. Stallknecht B, Dela F, Helge JW. Are blood flow and lipolysis in subcutaneous adipose tissue influenced by contractions in adjacent muscles in humans? Am. J. Physiol. Endocrinol. Metab. 292(2), E394-E399 (2007).

3. Crampes F, Beauville M, Riviere D, Garrigues M. Effect of physical training in humans on the response of isolated fat cells to epinephrine. J. Appl. Physiol. 61(1), 25-29 (1986).

4. Crampes F, Beauville M, Riviere D, Garrigues M, Lafontan M. Lack of desensitization of catecholamine-induced lipolysis in fat cells from trained and sedentary women after physical exercise. J. Clin. Endocrinol. Metab. 67(5), 1011-1017 (1988).

5. Ronn T, Volkov P, Tornberg A et al. Extensive changes in the transcriptional profile of human adipose tissue including genes involved in oxidative phosphorylation after a 6-month exercise intervention. Acta Physiol. 211(1), 188-200 (2014).

$\bullet$ Exercise induces extensive transcriptomic changes in human adipose tissue, notably in the expression of genes involved in oxidative phosphorylation and many metabolic pathways.

6. Ruschke K, Fishbein L, Dietrich A et al. Gene expression of PPARgamma and PGC-1alpha in human omental and subcutaneous adipose tissues is related to insulin resistance markers and mediates beneficial effects of physical training. Eur. J. Endocrinol. 162(3), 515-523 (2010).

7. Mulla NA, Simonsen L, Bulow J. Post-exercise adipose tissue and skeletal muscle lipid metabolism in humans: the effects of exercise intensity. J. Physiol. 524(Pt 3), 919-928 (2000).

8. Thompson D, Karpe F, Lafontan M, Frayn K. Physical activity and exercise in the regulation of human adipose tissue physiology. Physiol. Rev. 92(1), 157-191 (2012).

- Reviews the complex links between physical activity and adipose tissue function.

9. Cedar H, Bergman Y. Linking DNA methylation and histone modification: patterns and paradigms. Nat. Rev. Genet. 10(5), 295-304 (2009).

10. Barres R, Zierath JR. DNA methylation in metabolic disorders. Am. J. Clin. Nutr. 93(4), S897-S900 (2011).

11. Jacobsen MJ, Mentzel CM, Olesen AS et al. Altered methylation profile of lymphocytes is concordant with perturbation of lipids metabolism and inflammatory response in obesity. J. Diabetes Res. 2016, 8539057 (2016). 
12. Kirchner H, Nylen C, Laber S et al. Altered promoter methylation of PDK4, IL1 B, IL6, and TNF after Roux-en Y gastric bypass. Surg. Obes. Relat. Dis. 10(4), 671-678 (2014).

13. Simar D, Versteyhe S, Donkin I et al. DNA methylation is altered in B and NK lymphocytes in obese and Type 2 diabetic human. Metabolism 63(9), 1188-1197 (2014).

14. Ronn T, Volkov P, Davegardh C et al. A six months exercise intervention influences the genome-wide DNA methylation pattern in human adipose tissue. PLoS Genet. 9(6), e1003572 (2013).

-• Exercise induces genome-wide DNA methylation changes in human adipose tissue, potentially affecting adipocyte metabolism.

15. Kangaspeska S, Stride B, Metivier R et al. Transient cyclical methylation of promoter DNA. Nature 452(7183), 112-115 (2008).

16. Metivier R, Gallais R, Tiffoche $\mathrm{C}$ et al. Cyclical DNA methylation of a transcriptionally active promoter. Nature 452(7183), 45-50 (2008).

17. Barres R, Yan J, Egan B et al. Acute exercise remodels promoter methylation in human skeletal muscle. Cell Metab. 15(3), 405-411 (2012).

-• Acute exercise is associated with dynamic changes in DNA methylation profile in skeletal muscle suggesting that DNA hypomethylation is an early event in contraction-induced gene activation.

18. Pattamaprapanont P, Garde C, Fabre O, Barres R. Muscle contraction induces acute hydroxymethylation of the exercise-responsive gene Nr4a3. Front. Endocrinol. (Lausanne) 7, 165 (2016).

19. Nitert MD, Dayeh T, Volkov P et al. Impact of an exercise intervention on DNA methylation in skeletal muscle from first-degree relatives of patients with Type 2 diabetes. Diabetes 61(12), 3322-3332 (2012).

20. Denham J, Marques FZ, O’Brien BJ, Charchar FJ. Exercise: putting action into our epigenome. Sports Med. 44(2), 189-209 (2013).

-. Reviews the effect of physical (aerobic and resistance) exercise on epigenetic modifications in different tissues.

21. Horsburgh S, Robson-Ansley P, Adams R, Smith C. Exercise and inflammation-related epigenetic modifications: focus on DNA methylation. Exerc. Immunol. Rev. 21, 26-41 (2015).

22. Barres R, Gremeaux T, Gual P et al. Enigma interacts with adaptor protein with $\mathrm{PH}$ and $\mathrm{SH} 2$ domains to control insulin-induced actin cytoskeleton remodeling and glucose transporter 4 translocation. Mol. Endocrinol. 20(11), 2864-2875 (2006).

23. Egan B, Zierath JR. Exercise metabolism and the molecular regulation of skeletal muscle adaptation. Cell Metab. 17(2), 162-184 (2013).

24. Boyle P, Clement $\mathrm{K}, \mathrm{Gu} \mathrm{H}$ et al. Gel-free multiplexed reduced representation bisulfite sequencing for large-scale DNA methylation profiling. Genome Biol. 13(10), R92 (2012).

25. Donkin I, Versteyhe S, Ingerslev LR et al. Obesity and bariatric surgery drive epigenetic variation of spermatozoa in humans. Cell Metab. 23(2), 369-378 (2016).

26. Krueger F, Andrews SR. Bismark: a flexible aligner and methylation caller for Bisulfite-Seq applications. Bioinformatics 27(11), 1571-1572 (2011).

27. Langmead B, Salzberg SL. Fast gapped-read alignment with Bowtie 2. Nat. Methods 9(4), 357-359 (2012).

28. Hebestreit K, Dugas M, Klein HU. Detection of significantly differentially methylated regions in targeted bisulfite sequencing data. Bioinformatics 29(13), 1647-1653 (2013).

29. Andersen E, Ingerslev LR, Fabre $\mathrm{O}$ et al. Preadipocytes from obese humans with Type 2 diabetes are epigenetically reprogrammed at genes controlling adipose tissue function. Int. J. Obes. doi:10.1038/s41366-018-0031-3 (2018) (Epub ahead of print).

30. Karolchik D, Hinrichs AS, Furey TS et al. The UCSC Table Browser data retrieval tool. Nucleic Acids Res. 32, D493-D496 (2004).

31. Robinson MD, McCarthy DJ, Smyth GK. EdgeR: a bioconductor package for differential expression analysis of digital gene expression data. Bioinformatics 26(1), 139-140 (2010).

32. Ingerslev LR, Donkin I, Fabre $\mathrm{O}$ et al. Endurance training remodels sperm-borne small RNA expression and methylation at neurological gene hotspots. Clin. Epigenetics 10, 12 (2018).

33. Huang Da W, Sherman BT, Lempicki RA. Systematic and integrative analysis of large gene lists using DAVID bioinformatics resources. Nat. Protoc. 4(1), 44-57 (2009).

34. Huang Da W, Sherman BT, Lempicki RA. Bioinformatics enrichment tools: paths toward the comprehensive functional analysis of large gene lists. Nucleic Acids Res. 37(1), 1-13 (2009).

35. Egan B, O'Connor PL, Zierath JR, O'Gorman DJ. Time course analysis reveals gene-specific transcript and protein kinetics of adaptation to short-term aerobic exercise training in human skeletal muscle. PLoS ONE 8(9), e74098 (2013).

36. Astori G, Vignati F, Bardelli S et al. 'In vitro' and multicolor phenotypic characterization of cell subpopulations identified in fresh human adipose tissue stromal vascular fraction and in the derived mesenchymal stem cells. J. Transl. Med. 5, 55 (2007).

37. Bourin P, Bunnell BA, Casteilla L et al. Stromal cells from the adipose tissue-derived stromal vascular fraction and culture expanded adipose tissue-derived stromal/stem cells: a joint statement of the International Federation for Adipose Therapeutics and Science (IFATS) and the International Society for Cellular Therapy (ISCT). Cytotherapy 15(6), 641-648 (2013). 
38. Czepluch FS, Barres R, Caidahl K et al. Strenuous physical exercise adversely affects monocyte chemotaxis. Thromb. Haemost. 105(1), 122-130 (2011).

39. Weisberg SP, McCann D, Desai M, Rosenbaum M, Leibel RL, Ferrante AW Jr. Obesity is associated with macrophage accumulation in adipose tissue. J. Clin. Invest. 112(12), 1796-1808 (2003).

40. Xu H, Barnes GT, Yang Q et al. Chronic inflammation in fat plays a crucial role in the development of obesity-related insulin resistance. J. Clin. Invest. 112(12), 1821-1830 (2003).

41. Cancello R, Henegar C, Viguerie $\mathrm{N}$ et al. Reduction of macrophage infiltration and chemoattractant gene expression changes in white adipose tissue of morbidly obese subjects after surgery-induced weight loss. Diabetes 54(8), 2277-2286 (2005).

42. Curat $\mathrm{CA}$, Wegner $\mathrm{V}$, Sengenes $\mathrm{C}$ et al. Macrophages in human visceral adipose tissue: increased accumulation in obesity and a source of resistin and visfatin. Diabetologia 49(4), 744-747 (2006).

43. Frydelund-Larsen L, Akerstrom T, Nielsen S, Keller P, Keller C, Pedersen BK. Visfatin mRNA expression in human subcutaneous adipose tissue is regulated by exercise. Am. J. Physiol. Endocrinol. Metab. 292(1), E24-E31 (2007).

44. Galuska D, Kotova O, Barres R, Chibalina D, Benziane B, Chibalin AV. Altered expression and insulin-induced trafficking of $\mathrm{Na}+\mathrm{K}+$-ATPase in rat skeletal muscle: effects of high-fat diet and exercise. Am. J. Physiol. Endocrinol. Metab. 297(1), E38-E49 (2009).

45. Glund S, Treebak JT, Long YC et al. Role of adenosine $5^{\prime}$-monophosphate-activated protein kinase in interleukin- 6 release from isolated mouse skeletal muscle. Endocrinology 150(2), 600-606 (2009).

46. Hojbjerre L, Rosenzweig M, Dela F, Bruun JM, Stallknecht B. Acute exercise increases adipose tissue interstitial adiponectin concentration in healthy overweight and lean subjects. Eur. J. Endocrinol. 157(5), 613-623 (2007).

47. Holmes AG, Watt MJ, Febbraio MA. Suppressing lipolysis increases interleukin-6 at rest and during prolonged moderate-intensity exercise in humans. J. Appl. Physiol. 97(2), 689-696 (2004).

48. Keller C, Keller P, Marshal S, Pedersen BK. IL-6 gene expression in human adipose tissue in response to exercise-effect of carbohydrate ingestion. J. Physiol. 550(Pt 3), 927-931 (2003).

49. Keller P, Keller C, Steensberg A, Robinson LE, Pedersen BK. Leptin gene expression and systemic levels in healthy men: effect of exercise, carbohydrate, interleukin-6, and epinephrine. J. Appl. Physiol. 98(5), 1805-1812 (2005).

50. Leick L, Lindegaard B, Stensvold D, Plomgaard P, Saltin B, Pilegaard H. Adipose tissue interleukin-18 mRNA and plasma interleukin-18: effect of obesity and exercise. Obesity 15(2), 356-363 (2007).

51. Lyngso D, Simonsen L, Bulow J. Interleukin-6 production in human subcutaneous abdominal adipose tissue: the effect of exercise. $J$. Physiol. 543(Pt 1), 373-378 (2002).

52. Zouhal H, Jacob C, Delamarche P, Gratas-Delamarche A. Catecholamines and the effects of exercise, training and gender. Sports Med. 38(5), 401-423 (2008).

53. Benziane B, Burton TJ, Scanlan B et al. Divergent cell signaling after short-term intensified endurance training in human skeletal muscle. Am. J. Physiol. Endocrinol. Metab. 295(6), E1427-E1438 (2008).

54. Peake J, Nosaka K, Suzuki K. Characterization of inflammatory responses to eccentric exercise in humans. Exerc. Immunol. Rev. 11, 64-85 (2005).

55. Peake JM, Suzuki K, Wilson G et al. Exercise-induced muscle damage, plasma cytokines, and markers of neutrophil activation. Med. Sci. Sports Exerc. 37(5), 737-745 (2005).

56. Suzuki K, Totsuka M, Nakaji $S$ et al. Endurance exercise causes interaction among stress hormones, cytokines, neutrophil dynamics, and muscle damage. J. Appl. Physiol. 87(4), 1360-1367 (1999).

57. Mittal M, Siddiqui MR, Tran K, Reddy SP, Malik AB. Reactive oxygen species in inflammation and tissue injury. Antioxid. Redox Signal. 20(7), 1126-1167 (2014).

58. Perry CG, Lally J, Holloway GP, Heigenhauser GJ, Bonen A, Spriet LL. Repeated transient mRNA bursts precede increases in transcriptional and mitochondrial proteins during training in human skeletal muscle. J. Physiol. 588(Pt 23), 4795-4810 (2010).

-. Demonstrates that the mRNA response to exercise was attenuated with exercise training, as the muscle adapted to the exercise challenge.

59. Laker RC, Garde C, Camera DM et al. Transcriptomic and epigenetic responses to short-term nutrient-exercise stress in humans. Sci. Rep. 7(1), 15134 (2017).

60. Bouloumie A, Planat V, Devedjian JC et al. Alpha 2-adrenergic stimulation promotes preadipocyte proliferation. Involvement of mitogen-activated protein kinases. J. Biol. Chem. 269(48), 30254-30259 (1994).

61. De Glisezinski I, Moro C, Pillard F et al. Aerobic training improves exercise-induced lipolysis in SCAT and lipid utilization in overweight men. Am. J. Physiol. Endocrinol. Metab. 285(5), E984-E990 (2003).

62. Richterova B, Stich V, Moro C et al. Effect of endurance training on adrenergic control of lipolysis in adipose tissue of obese women. J. Clin. Endocrinol. Metab. 89(3), 1325-1331 (2004).

63. Luther J, Driessler F, Megges M et al. Elevated Fra-1 expression causes severe lipodystrophy. J. Cell Sci. 124(Pt 9), 1465-1476 (2011). 
64. Rao RR, Long JZ, White JP et al. Meteorin-like is a hormone that regulates immune-adipose interactions to increase beige fat thermogenesis. Cell 157(6), 1279-1291 (2014).

65. Chung HS, Hwang SY, Choi JH et al. Implications of circulating Meteorin-like (Metrnl) level in human subjects with Type 2 diabetes. Diabetes Res. Clin. Pract. 136, 100-107 (2018).

66. Redonnet A, Ferrand C, Bairras C et al. Synergic effect of vitamin A and high-fat diet in adipose tissue development and nuclear receptor expression in young rats. Br. J. Nutr. 100(4), 722-730 (2008).

67. Redonnet A, Bonilla S, Noel-Suberville C et al. Relationship between peroxisome proliferator-activated receptor gamma and retinoic acid receptor alpha gene expression in obese human adipose tissue. Int. J. Obes. Relat. Metab. Disord. 26(7), 920-927 (2002).

68. Abe T, Hirasaka K, Kagawa $S$ et al. Cbl-b is a critical regulator of macrophage activation associated with obesity-induced insulin resistance in mice. Diabetes 62(6), 1957-1969 (2013).

69. Vangaveti V, Shashidhar V, Jarrod G, Baune BT, Kennedy RL. Free fatty acid receptors: emerging targets for treatment of diabetes and its complications. Ther. Adv. Endocrinol. Metab. 1(4), 165-175 (2010).

70. Sica GL, Zhu G, Tamada K, Liu D, Ni J, Chen L. RELT, a new member of the tumor necrosis factor receptor superfamily, is selectively expressed in hematopoietic tissues and activates transcription factor NF-kappaB. Blood 97(9), 2702-2707 (2001). 